\title{
A Neuromorphic Silicon Photonics Nonlinear Equalizer For Optical Communications With Intensity Modulation and Direct Detection
}

\author{
Andrew Katumba ${ }^{\circledR}$, Student Member, IEEE, Xin Yin ${ }^{\circledR}$, Member, IEEE, Joni Dambre ${ }^{\circledR}$, Member, IEEE, \\ and Peter Bienstman ${ }^{\mathbb{1}}$, Member, IEEE
}

\begin{abstract}
We present the design and numerical study of a nonlinear equalizer for optical communications based on silicon photonics and reservoir computing. The proposed equalizer leverages the optical information processing capabilities of integrated photonic reservoirs to combat distortions both in metro links of a few hundred kilometers and in high-speed short-reach intensitymodulation-direct-detection links. We show nonlinear compensation in unrepeated metro links of up to $200 \mathrm{~km}$ that outperform electrical feedforward equalizers based equalizers, and ultimately any linear compensation device. For a high-speed short-reach 40$\mathrm{Gb} / \mathrm{s}$ link based on a distributed feedback laser and an electroabsorptive modulator, and considering a hard decision forward error correction limit of $0.2 \times 10^{-2}$, we can increase the reach by almost $10 \mathrm{~km}$. Our equalizer is compact (only 16 nodes) and operates in the optical domain without the need for complex electronic DSP, meaning its performance is not bandwidth constrained. The approach is, therefore, a viable candidate even for equalization techniques far beyond 100G optical communication links.
\end{abstract}

Index Terms-Neuromorphic computing, nonlinear equalization, reservoir computing, silicon photonics.

\section{INTRODUCTION}

$\mathbf{O}$ PTICAL technology is at the core of all modern telecommunication for high-speed, long-, medium- and shortreach applications. Fiber-based technologies are pervasive in all forms of networks from core and metro to data center and local area networks. Moreover, industry continually strives to get lightwave technology closer and closer to the last-mile end-user to take advantage of the massive bandwidth, energy efficiency and other benefits it provides. These advantages, combined with advances in photonics leading to cheaper network components (lasers, modulators, optical amplifiers), place lightwave technology at the core of today's internet information superhighway.

The industrial push for optical communications technology in access networks has in turn led to a surge in the demand for various intensely connected applications such as cloud services, video streaming and virtual reality. This strain on the end-user networks has predictably rippled back to all parts of the optical communication networks from metro to long haul in a race to squeeze out every last bit of capacity.

This is, however, no trivial undertaking, as the various elements constituting fiber-based lightwave networks also contribute to the degradation of optical signals during generation, transmission and reception phases [1]. In this work we propose an approach that can be applied to combat these unwanted signal modifications: it utilizes silicon photonics, a well-established CMOS-compatible technology, and photonic reservoir computing, a state-of-the-art brain-inspired information processing technology.

The taxonomy of fiber optic communication impairments consists of linear or nonlinear forms [2]. Linear impairments include Chromatic Dispersion (CD), Polarization Mode Dispersion (PMD), Symbol Timing Offset and Optical filtering. Nonlinear impairments include laser phase noise, Self Phase Modulation (SPM), Cross Phase Modulation (XPM), Four Wave Mixing (FWM) and nonlinear phase noise. In this work, as we are studying single-wavelength communication, XPM and FWM do not apply. Similarly, the inelastic nonlinearities of Stimulated Brillouin Scattering (SBS) and Stimulated Raman Scattering (SRS) are not considered in this work as they occur at power levels that are not of interest to the telecom links under consideration here.

Traditionally, linear imperfections in optical communication networks have been addressed with well-known techniques: optically, using dispersion compensation fibers and dispersion shifted fibers, and electrically, using tapped delay line based Feedforward Equalizers (FFEs) or Decision Feedback Equalizers (DFEs), or a combination of both.

The Kerr nonlinearity presents a fundamental limit to the scaling up of optical communication fiber capacity. Nonlinearity in fibers limits the maximum allowable SNR in optical 
communication links. As a result, numerous optical and digital nonlinearity mitigation techniques have been studied to surmount this limit. Digital Backpropagation (DBP), typically implemented on DSP chips, combats both linear and nonlinear impairments by solving the inverse NLSE to estimate the transmitted signal [3]. However, DBP is resource intensive and complex to implement for practical applications. Nonlinear Volterra series equalizers (V-NLEs) model fiber nonlinearities as a series of Volterra kernels which are then inverted to undo the distortions to the transmitted signal. V-NLEs are easier to implement and more computationally efficient than DBP, especially for intrachannel applications [4], but are still quite resource-demanding. Commercially, these two DSP-based nonlinear compensation techniques dominate all other forms. Optically, nonlinearity mitigation has successfully been demonstrated using optical phase conjugation (OPC), where a single optoelectronic component is inserted in the center of the transmission line to invert the polarity of the dispersion and nonlinearity parameters from the first phase of the transmission [5], such that they are compensated for in the 2nd part of the link. However, the placement of the OPC node limits network design flexibility and puts restrictions on the expected dispersion and power evolution profiles. Phase-conjugated twin wave (PCTW) is a more recent technique that offers a low complexity (simpler DSP), and is an effective solution for optical nonlinearity mitigation [6]. It is however coupled to reduced spectral efficiency (SE) because of the extra overhead to transmit the phase conjugated copy of the transmitted signal. Another set of digital approaches for nonlinear compensation is based on machine learning techniques. Some popular implementations are based on Artificial Neural Networks (ANNs) ([7]) and Support Vector Machines (SVMs) ([8], [9]). In [10] techniques based on nonlinear state-space based Bayesian filtering and Gaussian Mixture Models (GMMs) are presented. A more recent study of a photonic machine learning implementation for signal recovery in optical communications can be found in [11].

The advent of coherent optical communications for $100 \mathrm{G}$ and beyond has further pushed the reliance on DSP technology as these networks almost exclusively rely on the DSP for their linear and nonlinear impairment mitigation, as well as a plethora of other receiver-side post-processing tasks. Lately, the same DSPs (typically with some reduction in complexity) have been used to implement various functions in IM-DD links for highspeed short reach-applications involving PAM-4 and DMT such as in [12] and [13]. But, as we alluded to earlier, DSPs are notoriously power hungry and their design complexity has been growing steadily, especially in a bid to keep the wall plug efficiency within manageable limits. As a consequence, there is a lot of interest in solutions that seek to either entirely replace DSP functions (DSP-less) or simplify the complexity of the task that has to be solved by the DSP chip for both coherent and IM-DD optical communications systems.

In this paper, we propose a novel signal equalizer to undo both impairments in optical communication networks based on reservoir computing and study its performance in IM-DD links. Our approach operates in the optical domain meaning, that it is very fast, compact (only 16 nodes on a footprint of $\approx 16 \mathrm{~mm}^{2}$ ) and energy efficient. Additionally being CMOS-compatible means that we can take advantages of CMOS mass production benefits for scaling. Moreover it can easily be co-integrated with receiver electronics via monolithic photonic-electronic co-integration in, for example, a transceiver module.

The rest of this paper is structured as follows. In Section II, we describe reservoir computing in more detail. Then, in Section III, we explain the general simulation setup, training details and machine learning techniques used in the study. This is followed by a presentation and discussion of results for metro and shortreach applications in Section IV. We provide a summary of results and a conclusion in Section V.

\section{Photonic Reservoir Computing}

Reservoir Computing (RC) is a brain-inpired non-VonNeumann analog computing approach that relies on the information processing capabilities of certain physical systems [14] [16]. Initially invented as a software technique for processing temporal data on digital computers, reservoir computing has evolved into a computationally versatile platform capable of tackling a wide range of tasks. It excels at tackling tasks that involve sequential data such as speech recognition and time series prediction. A key extension to reservoir computing is implementing the reservoir in hardware [17]. Examples of RC implemented in mechanical systems, memristive systems, atomic switch networks, boolean logic elements and photonics as substrates can be found in [18]-[22].

The RC system consists of three basic parts: an input layer which couples the input signal into a nonlinear dynamical system, "the reservoir" (i.e. the recurrent neural network, which is kept untrained) and finally the output layer that typically linearly combines the states of the reservoir to provide the timedependent output signal [17]. An illustration of this architecture is given in Fig. 1.

The way to apply the RC approach to solve a particular task typically takes the form of setting it up as a supervised machine learning (linear regression) problem. Known labeled data (training examples) is used to train the weights to compute the output (the readout) that is typically a linear combination of the recorded signals at each reservoir node. These weights are then stored and used to generate the output signal for future input signal sequences. RC systems are fast to train and have shown state-of-the-art performance on time-dependent data (such as speech recognition, nonlinear channel equalization, robot control, time series prediction, financial forecasting, handwriting recognition, etc.) on a range of complex tasks [17].

Experimental demonstrations of photonic reservoirs routinely achieve state-of-the-art performance on various information processing tasks. Implementations based on a single nonlinear node with a delayed feedback architecture can be found in [23]-[28], while integrated photonic reservoirs have been investigated experimentally and numerically in [21], [29]-[32].

Passive photonic reservoirs are a more recent invention in which the input signal propagates through a passive linear 


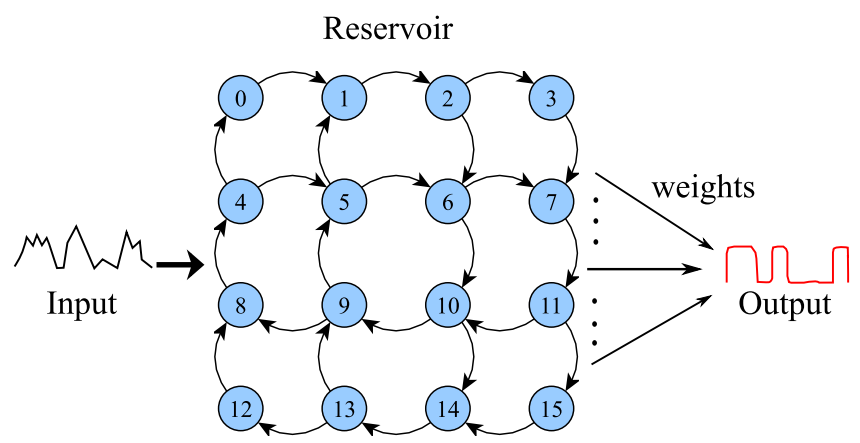

Fig. 1. Layout of the photonic reservoir computing setup as is used in this work for impairment removal. The input signal is a distorted non-return-to-zero onoff-keying (NRZ-OOK) signal that has traversed a fiber optic link, the integrated photonics reservoir is composed 16 nodes arranged in a swirl topology and the reservoir states are recorded at each node with aid of a photodetector. These states are then recorded and used to train a set of weights that represent the readout function that generates the final output signal. Note that in the reservoir, the numbered lightlight blue circles $\left(n_{i}\right)$ are the nodes in the reservoir and in this architecture are the locations where states are combined and split, and also serve as the input and detection points.

photonic network, i.e., one without amplification or nonlinear elements. The required nonlinearity is introduced at the readout point, typically with a photodetector [23], [32]. The photonic reservoir computing equalizer in this work is based on the passive integrated reservoir design that was introduced in [32]. Apart from simplicity from a fabrication point-of-view, a further advantage of such a passive architecture is the reduced power consumption, since the computation itself does not require external energy. As in [32], the reservoir nodes are laid out in a swirl topology to satisfy planarity constraints of the CMOS SOI platform while allowing for a reasonable mixing of the input signals. Physically, the linear network part of the reservoir considered in this work is composed of delay lines made up of strip waveguides (in actual designs there are sometimes folded into spiral shape for compactness) interconnected by Multimode Interference (MMI) couplers, all well-known robust components ubiquitous in integrated photonic circuits. Note that we define the interconnection delay as the time it takes to for light to propagate between any two nodes (or to travel across any delay line). A schematic of 16-node photonic swirl reservoir is shown in Fig. 1.

In discretized time, the passive reservoir state update equation can be generalized as:

$$
\vec{x}[k+1]=\boldsymbol{W}_{\text {res }} \vec{x}[k]+\vec{w}_{i n}\left(\vec{u}[k+1]+u_{\text {bias }}\right)
$$

where $\vec{u}$ is the input to the reservoir and $u_{\text {bias }}$ is a fixed scalar bias applied to the inputs of the reservoir. For an N-node reservoir, $\boldsymbol{W}_{\text {res }}$ is an $N \times N$ matrix representing the interconnections between reservoir components taking into account splitting ratios and losses, with phases drawn from a random uniform distribution on $[-\pi, \pi], U(-\pi, \pi) \cdot \vec{w}_{i n}$ is an $N$-dimensional column vector whose elements are nonzero for each active input node. These input weights are similarly chosen from $U(-\pi, \pi)$ [33].

Another way of looking at the system described by Eq. (1) is that the reservoir acts as a non-linear filter which preprocesses the data such that it gets transformed into a higher-dimensional space, where the signals can be more easily classified using a linear classifier. Indeed, injecting a signal in the input port of the reservoir will result at each of the nodes in a complicated interferometric mixing of copies of the input signal with different intensities and different delays corresponding to the different paths in the reservoir between the input and that output node. These signals are then non-linearly coupled through the action of the detector, which transforms complex-valued amplitudes into real-valued intensities. By taking suitable linear combinations of the signals at each node, the system can be trained in such a way as to minimise the difference between the output of the reservoir and the original, unperturbed bitstream at the beginning of the link. More details about reservoir computing can be found in [32].

Our previous work in [32] experimentally verifies that a passive integrated photonic reservoir can yield error-free performance on the header recognition task for headers up to 3 bits in length, as well as on a number of digital optical bit level manipulations that could be useful for various telecommunications tasks such as parity, coding, etc.

Here, we present an equalizer implemented using an integrated photonics reservoir computing (PhRC) system. Conceptually, the PRhC equalizer is a high-dimensional complex nonlinear filter that acts in the optical domain to undo distortions. As the the reservoir has memory, it combines delayed versions of the input signal (similar to a tapped filter) and additionally performs a nonlinear transformation of the input signal (which is necessary for creating rich nonlinear combinations of delayed versions of the input signal) that can be used to combat distortions such as those induced by SPM and CD.

The integrated $\mathrm{PhRC}$ equalizer will naturally benefit from the advantages of the CMOS platform in terms of scalability and energy efficiency. The design is flexible and can be applied to virtually all sorts of links without limit in terms of required bandwidth. Designing a $40 \mathrm{~Gb} / \mathrm{s}$ or a $400 \mathrm{~Gb} / \mathrm{s}$ equalizer is a matter of changing the internal timescale of the reservoir (set by the length of the delay lines); the ultimate limit lies in the readout electronics, making the PhRC equalizer a future-proof option to signal equalization. Once deployed, the equalizer can easily be retrained in the field (on demand or on a schedule) to adapt to the link conditions. The PhRC equalizer could also be deployed just before a DSP to reduce the computational load associated with cleaning up severely distorted signals. We will show numerical simulations of the equalizer operating under various conditions for realistic fibre optical links using the resulting Bit Error Error Rate as the performance metric.

\section{Simulation Setup AND Methodology}

We focus on designing PhRC equalizers for metro and shortreach optical communications links. The optical communication links were set up in VPI Transmission Maker v9.8 software. The setup used for the metro link is given in Figure 2 while for the high-speed short-reach links Figure 7 was used. VPI software incorporates a wealth of validated models of transmitter and 


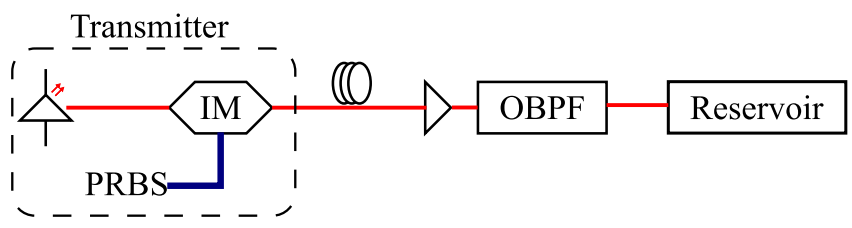

Fig. 2. Schematic representation of the simulation setup to generate data for the signal equalization task. The input pseudo-random bit sequence (PRBS) signal is modulated onto a laser signal, transmitted over a fiber link, amplified and filtered, after which the field of the optical signal is saved to file to be used as input to the photonic reservoir simulation model. OBPF - Optical band pass filter.

TABLE I

Key PARAMETERS For SSMF USED In TRANSMISSION SIMULATIONS

\begin{tabular}{ccc}
\hline Parameter & Value & Units \\
\hline Attenuation & 0.2 & $\mathrm{~dB} / \mathrm{km}$ \\
Dispersion Coefficient & $16 \times 10^{-6}$ & $\mathrm{~s} / \mathrm{m}^{2}$ \\
Dispersion Slope & $0.08 \times 10^{3}$ & $\mathrm{~s} / \mathrm{m}^{3}$ \\
Nonlinear Index & $2.6 \times 10^{-20}$ & $\mathrm{~m}^{2} / \mathrm{W}$ \\
Core Area & $80.0 \times 10^{-12}$ & $\mathrm{~m}^{2}$ \\
\hline
\end{tabular}

receiver components as well as implementations of all signal degradation mechanisms encountered as signals traverse optical telecom links. Standard single-mode fiber (SSMF) was used in all cases with parameters given in Table I.

After traversing the link, the electric field of the signal was recorded and saved offline, to be processed by the numerical circuit level model of the passive integrated photonics reservoir. In an experimental setup, this coupling from the fiber into the reservoir chip could for example be achieved using fiber-tochip grating couplers. We should mention that, as we operate in a single polarization (Transverse Electric (TE) in this case), a polarization controller component is inserted just before the reservoir chip to ensure maximum transmission. The simulation model was built with custom time-domain circuit simulation scripts based on the Caphe software [34]. In all cases a $4 \times 4$ (16-node) reservoir architecture was used to generate the states. This number of nodes was chosen as it yields a design that is cost-effective to produce with multi-project wafer runs, and has achieved good performance on a number of tasks [32], as well as the impact of other model and training parameters, on the performance of the PhRC equalizer will further be discussed in subsequent sections.

The states recorded from each reservoir node were transformed by a receiver model to take into account various noise contributions and bandwidth limitations. The specific model used is for a PIN photodetector with responsivity $1.0 \mathrm{~A}$ and thermal noise of $3.0 \times 10^{-12} \mathrm{~A} / \sqrt{\mathrm{Hz}}$. It has a 3rd order low pass Bessel electrical bandpass filter (ELBF) with cut-off frequency corresponding to 0.7 times the bit rate of the input signal. Shot noise in the PIN photodetector was accounted for as well.

After the receiver model, the states were then collated and used to train the readout weights using the scikit-learn library [35]. For each run, 10,000 randomly chosen input PRBS7 bits
TABLE II

TransmitTer Parameters For MEtro LinK SETUP

\begin{tabular}{ccc}
\hline Parameter & Value & Units \\
\hline & Laser & \\
Emmision Wavelength & 1550 & $\mathrm{~nm}$ \\
& Modulator & \\
Symmetry factor & -1 & - \\
Extinction Ratio & 20 & $\mathrm{~dB}$ \\
Rise time & $0.4 \times$ bit period & - \\
\hline
\end{tabular}

were used and the resulting states (after the receiver) were used for training with 5-fold cross-validation to optimize the model hyperparameters. Note that for each 1,000 bits a different random seed was used for the PRBS signal. The regularized ridge regression algorithm was used to train a linear readout. The reported bit error rate (BER) is the average validation error across folds. This process was repeated 8 times for different random initializations of the link conditions and reservoir phases. For each training run, the internal reservoir phases and the input phases are randomly generated and fixed.

The BER was calculated using VPI based on Gaussian statistics and optimization of the sampling instant. To calculate the BER error measure used in the readout training, the received signal is first sampled at a sampling instant (the optimal sampling instant is determined by simply checking at which of the samples the minimum error occurs).

\section{RESUlts AND DisCUSSION}

\section{A. Metro Links}

In this section, we investigate the performance of the PhRC equalizer on unrepeated fiber optic communications links in the metro regime for fiber lengths ranging from $100 \mathrm{~km}$ to $250 \mathrm{~km}$ for a $10 \mathrm{~Gb} / \mathrm{s}$ NRZ OOK link. The setup is shown as in Figure 2. Unless stated otherwise, in this section we assumed a launch power of $5 \mathrm{~mW}$ (after the modulator). The amplifier, with a noise figure (NF) of 4.0 (the same amplifier is used throughout the work), is set to entirely undo the link attenuation and the filter gets rid of out-of-band noise and is a 3rd order Bessel filter with bandwidth 4 times the data rate. Note that this data rate is chosen here to keep it compatible with the measurement capability in our characterization lab but the same procedure can be followed for higher speed links.

First, we study the influence of the node interconnection delay time on the BER of the PhRC equalizer. The node interconnection delay is the most important parameter for passive planar integrated photonics reservoirs as it determines the timescale at which signals interfere within the reservoir. It is set by changing the length on the on-chip delay lines at design time. The transmitter (consisting of the pattern generator, continuous wave (CW) laser and external Lithium Niobate modulator) parameters are given in Table II. The symmetry factor is an indicator of the chirp behaviour of the modulator and is a measure of the imbalance of the drive-induced phase shifts in its two arms. 


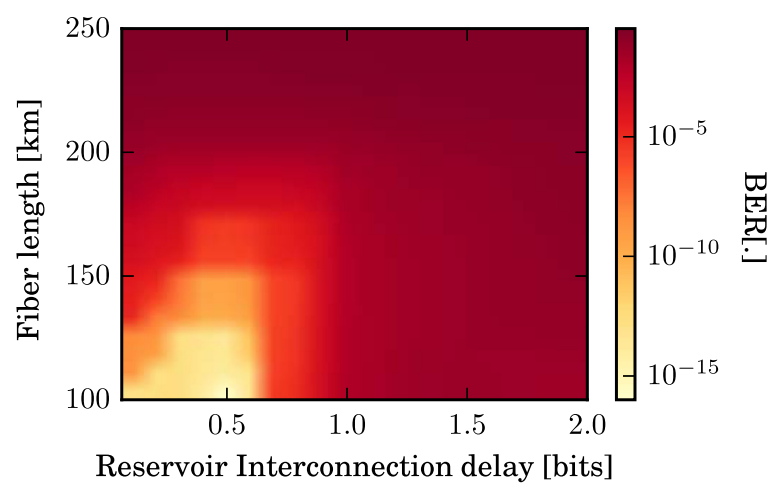

Fig. 3. Error rates vs reservoir interconnection delay and fiber length for 16 node PhRC equalizer for an NRZ-OOK link at $10 \mathrm{~Gb} / \mathrm{s}$.

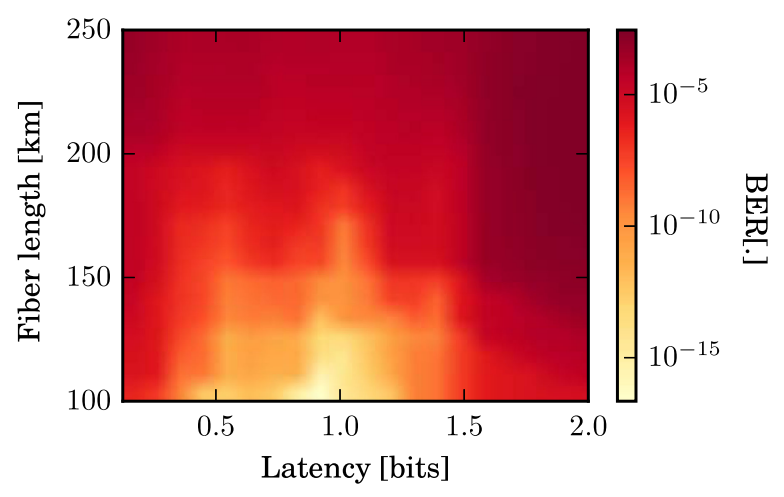

Fig. 4. Error rate vs latency and fiber length for a reservoir with interconnection delay time equal to half the bit duration.

A value of -1 means ideal intensity modulation with no chirp, meaning that the two drive voltages are equal in magnitude but out of phase.

As seen in Figure 3, the regime of best operation of the PhRC equalizer corresponds to an interconnection length that corresponds to a delay of half the bit duration. This result demonstrates that under these link conditions, the condition of the optimal interconnection delay coincides with those for the header recognition and bit level tasks of [32]. For the rest of the discussions in this section we therefore fix the interconnection length of the reservoir to this value.

As in any causal system, the reservoir output requires a finite time before responding to the input. We therefore need to find the delay between the input and the corresponding desired output for which the reservoir can best solve the task. This delay is termed latency in this work (we assume here that there are no external, more rigid latency constraints to take into account). In the interconnection delay studies above (as illustrated in Figure 3), we arbitrarily set the value of the latency to 1 bit duration. This turns out to be a good choice: Figure 4 shows that the latency has a significant impact on the performance of the reservoir (more than $10 \mathrm{~dB}$ difference in BER for most fiber lengths). The best latency depends on the fiber length, but is usually close to one bit period.

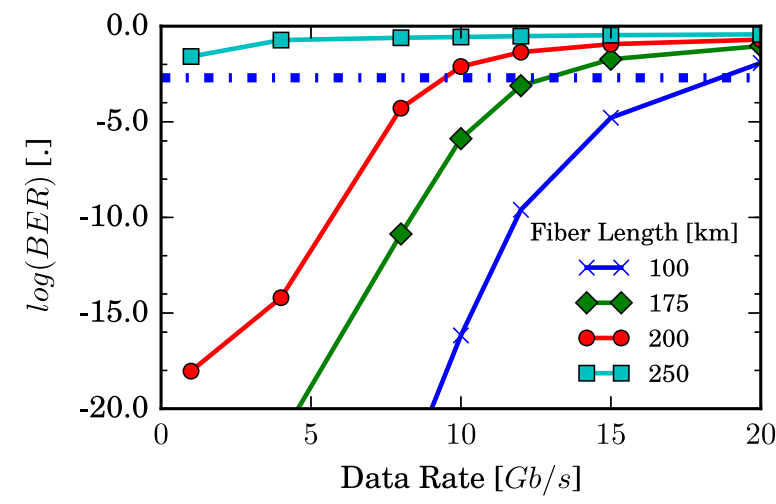

Fig. 5. Error rate vs data rate for a reservoir with interconnection delay time equal to half the bit duration and latency 1 bit. A Hard Decision Forward Error Correction limit (HD-FEC limit) of $0.2 \times 10^{-2}$ is also shown (dashed blue line). Error free operation is possible for all error rate values below this limit.

For all subsequent simulations in this section, we therefore set the reservoir inter-node delay time to half the data rate and the latency to 1 bit duration.

We then investigate the performance of this reservoir design for equalization for links running at different data rates. The results, given in Figure 5, show that for link lengths less than $200 \mathrm{~km}$ in length, the $\mathrm{PhRC}$ equalizer can operate at data rates higher than the design data rate of $10 \mathrm{~Gb} / \mathrm{s}$. For example for the $100 \mathrm{~km}$ link, we get error rates below the FEC limit for data rates close to $20 \mathrm{~Gb} / \mathrm{s}$. This result gives a measure of robustness of this particular PhRC equalizer. When designing equalizers for links with higher bit rates, the analysis would need to be repeated to find the best operating parameters (most notably the interconnection delay) for the equalizer.

Finally, we compare the performance of the PhRC equalizer for a link with the launch power changed to $15 \mathrm{~mW}$ (from the $5 \mathrm{~mW}$ of the previous simulations) to an FIR FFE filter trained on the same amount of data. An adaptive FFE filter with 31 taps is used (The filter goes over the training data four times to allow for convergence). The results are shown in Figure 6. The PhRC equalizer outperforms the FFE equalizer with BERs over 5 orders of magnitude lower at, for example, $150 \mathrm{~km}$ and an order or two of magnitude lower at $200 \mathrm{~km}$. The difference in performance originates in the fact that the PhRC equalizer is a nonlinear compensation device; it takes advantage of the nonlinear transformation in the reservoir to better model the distortion and outstrip the performance of the FFE filter. In Figure 6 we also plot the cases with and without the fiber nonlinearity.

We observe that at the distances under consideration, the fiber nonlinearities are not yet deleterious, and in fact performance for the FFE equaliser improves in some cases. While this may seem surprising, similar effects owing to the interplay of nonlinearities and dispersion have been observed before [36]. We do however observe that the reservoir is able to make use of its nonlinear nature to outperform the FFE equalizer for these links, i.e. the nonlinear compensator (the reservoir) is able to exploit these effects to a much higher degree than the linear 


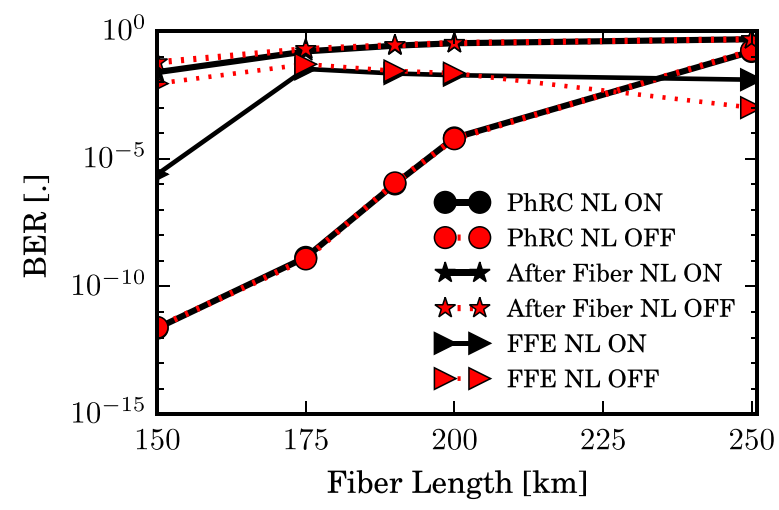

Fig. 6. BER of the PhRC equalizer as compared to that of an FIR Feed Forward Equalizer (FFE) trained on the same amount of data for different fiber lengths. The launch power is set to $15 \mathrm{~mW}$. NL ON- nonlinear propagation. NL OFF nonlinear propagation is deactivated (Nonlinear Index of the fiber in Table I is set to 0$)$.

compensator (the FFE). It is also interesting that the reservoirs performance is the same when the non-linearities are turned on or off. Its worth mentioning that in both cases, the weights used in the reservoir are different, which will result in the equaliser being in a different dynamical regime. The fact that ultimately these two reservoirs give similar performance, means that this performance limit is determined by the properties of reservoir computing, and not so much affected by the details of the nonlinear effects present in the channel. This is an important benefit, because it means that the same architecture (after retraining the weights) is very robust against variations in the details of the underlying transmission system.

\section{B. High-Speed Short-Reach Links}

We now investigate the applicability of the PhRC equalizer to high-speed short-reach IM/DD applications. EAMs are particularly suitable for high-speed short-reach communication links as they are low-cost and compact compared to external $\mathrm{LiNbO}_{3}$ modulators, can be designed to have relatively low chirp (for example when compared to directly modulated DFB lasers) and can be driven with lower driving voltage amplitudes. They can additionally be monolithically integrated on the same chip as a DFB continuous wave (CW) laser to yield a compact transmitter module - so called electroabsorption modulatorintegrated distributed-feedback (EADFB) lasers. EADFB lasers with speeds over $50 \mathrm{~Gb} / \mathrm{s}$ were demonstrated in [37] and are competitive candidates for the next beyond $400 \mathrm{~Gb} / \mathrm{s}$ optical communication links (multilane).

We set up a $40 \mathrm{~Gb} / \mathrm{s}$ link using a CW DFB laser and an EAM with parameters given in Table III. The input PRBS signal is then propagated for distances ranging from $1 \mathrm{~km}$ to $25 \mathrm{~km}$. Just as in the previous section, we perform a search for the optimum reservoir interconnection length and find that it lies somewhere between $20 \%$ and $50 \%$ of the bit duration. We assume a latency of 1 bit duration for the readout training phase as before.

The results for the BER obtained with the PhRC equalizer for the different fiber lengths are given in Figure 9 and show that
TABLE III

TransmitTer Parameters FOR High-SPEed SHORT-ReACH LinK SETUP

\begin{tabular}{ccc}
\hline Parameter & Value & Units \\
\hline & Laser & \\
Output Power & 10 & $\mathrm{~mW}$ \\
Emmision Wavelength & 1550 & $\mathrm{~nm}$ \\
& EAM & \\
Driver Voltage & 2.6 & $\mathrm{~V}$ \\
Driver Bias & -3 & $\mathrm{~V}$ \\
& EAM & \\
Transmission characteristics $(1550 \mathrm{~nm})$ & see Figure 8 & - \\
\hline
\end{tabular}

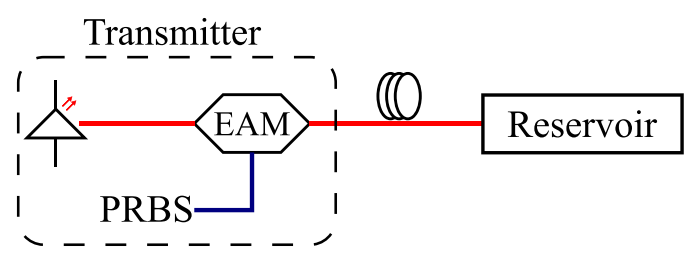

Fig. 7. Schematic representation of the setup used for short reach simulations The Mach-Zehnder Modulator of Figure 2 is replaced by an Electro-Absorptive Modulator. The EDFA at the end of the link has also been removed.

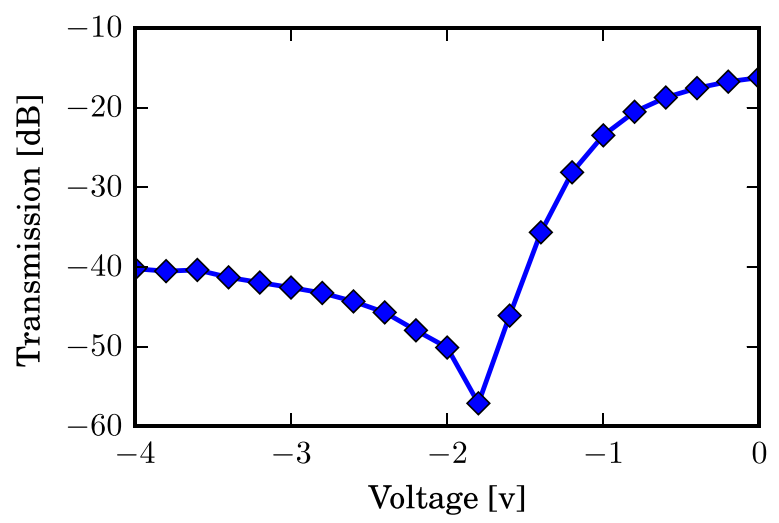

Fig. 8. Voltage-dependent transmission of the EAM used for the high speed short reach link at $1550 \mathrm{~nm}$.

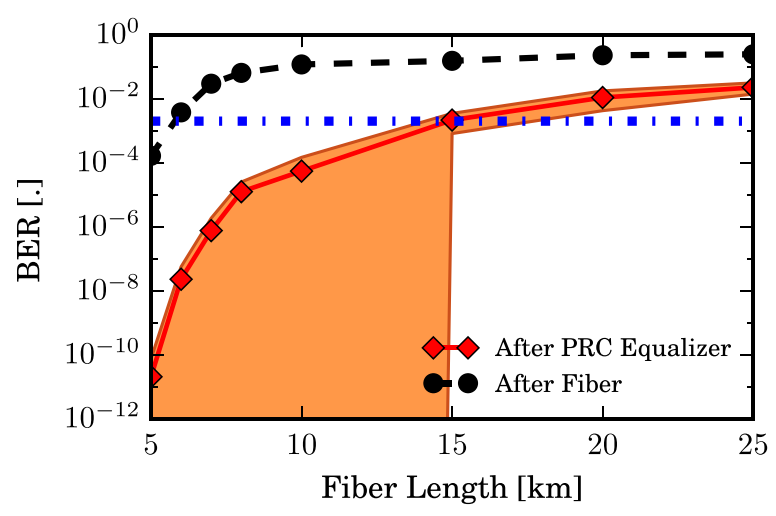

Fig. 9. BER vs fiber length for $40 \mathrm{~Gb} / \mathrm{s}$ short reach access link with a DFB and an EAM. The blue dashed line indicates the HD-FEC limit and the the filled section demarcates the error margin. 
we can triple the reach of links from $5 \mathrm{~km}$ to $15 \mathrm{~km}$ by using the $\mathrm{PhRC}$ equalizer compared to the case of no equalization.

\section{CONCLusion}

In this paper, we have presented a design for an integrated photonics reservoir equalizer that can undo imperfections in optical communication links. We have numerically studied the performance of this PhRC equalizer for two different types of IM/DD links: metro and high-speed short-reach. We can reach below the SD-FEC limit for metro links up to $200 \mathrm{~km}$ and we can extend the reach for a high-speed short-reach link running at $40 \mathrm{~Gb} / \mathrm{s}$ with an EAM from $5 \mathrm{~km}$ to $15 \mathrm{~km}$. In the future, we expect this task will serve as an important experimental benchmark for our integrated photonics reservoirs targeted at optical communication tasks.

In the short run, we also plan to extend this analysis to other IM/DD-compatible modulation formats such as DMT, duobinary and DPSK. Subsequently, we will verify the performance of the device on chips that are currently being fabricated.

\section{REFERENCES}

[1] I. Djordjevic, W. Ryan, and B. Vasic, Coding For Optical Channels. Boston, MA, USA: Springer, 2010.

[2] E. Ip and J. M. Kahn, "Compensation of dispersion and nonlinear impairments using digital backpropagation," J. Lightw. Technol., vol. 26, no. 20, pp. 3416-3425, Oct. 2008.

[3] D. Rafique, J. Zhao, and A. D. Ellis, "Digital back-propagation for spectrally efficient WDM 112 Gbit/s PM m-ary QAM transmission," Opt. Express, vol. 19, no. 6, pp. 5219-5224, 2011.

[4] L. Liu et al., "Intrachannel nonlinearity compensation by inverse Volterra series transfer function," J. Lightw. Technol., vol. 30, no. 3, pp. 310-316, Feb. 2012.

[5] D. Rafique, "Fiber nonlinearity compensation: Commercial applications and complexity analysis," J. Lightw. Technol., vol. 34, no. 2, pp. 544-553, Jan. 2016.

[6] X. Liu, A. R. Chraplyvy, P. J. Winzer, R. W. Tkach, and S. Chandrasekhar, "Phase-conjugated twin waves for communication beyond the Kerr nonlinearity limit," Nature Photon., vol. 7, no. 7, pp. 560-568, 2013.

[7] M. Jarajreh et al., "Artificial neural network nonlinear equalizer for coherent optical OFDM," IEEE Photon. Technol. Lett., vol. 27, no. 4, Feb. 2015, Art. no. 387-390.

[8] Y. Han, S. Yu, M. Li, J. Yang, and W. Gu, "An SVM-based detection for coherent optical APSK systems with nonlinear phase noise," IEEE Photon. J., vol. 6, no. 5, Oct. 2014, Art. no. 7100510.

[9] E. Giacoumidis et al., "Comparison of DSP-based nonlinear equalizers for intra-channel nonlinearity compensation in coherent optical OFDM," Opt. Lett., vol. 41, no. 11, pp. 2509-2512, 2016.

[10] D. Zibar, M. Piels, R. Jones, and C. G. Schaeffer, "Machine learning techniques in optical communication," J. Lightw. Technol., vol. 34, no. 6, pp. 1442-1452, Mar. 2016.

[11] A. Argyris, J. Bueno, and I. Fischer, "Photonic machine learning implementation for signal recovery in optical communications," Sci. Rep., vol. 8, no. 1,2018 , Art. no. 8487

[12] Z. Xian et al., "PDM PAM-4 with IM-DD using a simple MIMO DSPbased receiver for short reach communications," in Proc. Asia Commun. Photon. Conf., 2015, Paper AM3E.3.

[13] X. Zhou et al., "Polarization-multiplexed DMT with IM-DD using $2 \times 2$ MIMO processing based on sop estimation and MPBI elimination," IEEE Photon. J., vol. 7, no. 6, Dec. 2015, Art. no. 7802812.

[14] W. Maass, T. Natschläger, and H. Markram, "Real-time computing without stable states: A new framework for neural computation based on perturbations," Neural Comput., vol. 2560, pp. 2531-2560, 2002.

[15] H. Jaeger and H. Haas, "Harnessing nonlinearity: Predicting chaotic systems and saving energy in wireless communication," Science, vol. 304, pp. 78-80, 2004.
[16] D. Verstraeten, B. Schrauwen, M. D'Haene, and D. Stroobandt, "An experimental unification of reservoir computing methods," Neural Netw., vol. 20, no. 3, pp. 391-403, 2007.

[17] A. Katumba et al., "Neuromorphic computing based on silicon photonics and reservoir computing," IEEE J. Sel. Topics Quantum Electron., vol. 24, no. 6, Nov./Dec. 2018, Art. no. 8300310

[18] H. Hauser, A. Ijspeert, R. Füchslin, R. Pfeifer, and W. Maass, "Towards a theoretical foundation for morphological computation with compliant bodies," Biol. Cybern., vol. 105, no. 2011, pp. 355-370, 2011.

[19] H. O. Sillin et al., "A theoretical and experimental study of neuromorphic atomic switch networks for reservoir computing," Nanotechnology, vol. 24, 2013, Art. no. 384004

[20] M. S. Kulkarni and C. Teuscher, "Memristor-based reservoir computing," in Proc. IEEE/ACM Int. Symp. Nanoscale Archit., 2012, pp. 226-232.

[21] K. Vandoorne, "Photonic reservoir computing with a network of coupled semiconductor optical amplifiers," Ph.D. dissertation, Dept. Info. Technol. (INTEC), Ghent Univ., Ghent, Belgium, 2011.

[22] Y. Paquot et al., "Optoelectronic reservoir computing," Sci. Rep., vol. 2, 2012, Art. no. 287.

[23] Q. Vinckier et al., "High-performance photonic reservoir computer based on a coherently driven passive cavity," Optica, vol. 2, no. 5, pp. 438-446, 2015 .

[24] D. Brunner, M. C. Soriano, C. R. Mirasso, and I. Fischer, "Parallel photonic information processing at gigabyte per second data rates using transient states," Nature Commun., vol. 4, 2013, Art. no. 1364.

[25] L. Appeltant et al., "Information processing using a single dynamical node as complex system," Nature Commun., vol. 2, 2011, Art. no. 468.

[26] L. Larger et al., "Photonic information processing beyond Turing: An optoelectronic implementation of reservoir computing," Opt. Express, vol. 20, no. 3, p. 3241-3249, 2012.

[27] F. Duport, B. Schneider, A. Smerieri, M. Haelterman, and S. Massar, "Alloptical reservoir computing," Opt. Express, vol. 20, no. 20, pp. $22783-$ $22795,2012$.

[28] K. Hicke, M. Escalona-Morán, D. Brunner, M. C. Soriano, I. Fischer, and C. R. Mirasso, "Information processing using transient dynamics of semiconductor lasers subject to delayed feedback," IEEE J. Sel. Topics Quantum Electron., vol. 19, no. 4, Jul./Aug. 2013, Art. no. 1501610.

[29] C. Mesaritakis, V. Papataxiarhis, and D. Syvridis, "Micro ring resonators as building blocks for an all-optical high-speed reservoir-computing bit-pattern-recognition system," J. Opt. Soc. Amer. B, vol. 30, 2013, Art. no. 3048.

[30] M. A. A. Fiers et al., "Nanophotonic reservoir computing with photonic crystal cavities to generate periodic patterns," IEEE Trans. Neural Netw. Learn. Syst., vol. 25, no. 2, pp. 344-355, Feb. 2014.

[31] H. Zhang et al., "Integrated photonic reservoir computing based on hierarchical time-multiplexing structure," Opt. Express, vol. 22, no. 25, pp. 31356-31370, 122014

[32] K. Vandoorne et al., "Experimental demonstration of reservoir computing on a silicon photonics chip," Nature Commun., vol. 5, 2014, Art. no. 3541.

[33] A. Katumba, M. Freiberger, P. Bienstman, and J. Dambre, "A multipleinput strategy to efficient integrated photonic reservoir computing," Cogn. Comput., vol. 9, pp. 307-314, 2017.

[34] M. Fiers et al., "Time-domain and frequency-domain modeling of nonlinear optical components at the circuit-level using a node-based approach," J. Opt. Soc. Amer. B, vol. 29, no. 5, pp. 896-900, 2012.

[35] F. Pedregosa et al., "Scikit-learn: Machine learning in $\{\mathrm{P}\}$ ython," J. Mach. Learn. Res., vol. 12, pp. 2825-2830, 2011

[36] E. Information, "Digital chromatic dispersion pre-management enabled single-lane $112 \mathrm{~Gb} / \mathrm{s}$ PAM-4 signal transmission over $80 \mathrm{~km}$ SSMF," Opt. Lett., vol. 43, no. 7, pp. 1495-1498, 2018.

[37] T. Fujisawa et al., "Ultra-high-speed electroabsorption modulator integrated lasers for $100 \mathrm{GbE}$ and beyond," in Proc. Tech. Dig.-17th OptoElectron. Commun. Conf., 2012, pp. 75-76.

Andrew Katumba (S'14) was born in Masaka, Uganda, in 1985. He received the M.Sc. degree in optics and photonics from Karlsrule Institute of Technology, Karlsruhe, Germany, in 2013. He is currently working toward the Ph.D. degree in photonics engineering with the Photonics Research Group, Gent Universityimec, Gent, Belgium. His current research focuses on photonic neuromorphic architectures for high-speed optical telecommunications systems. He is a student member of IEEE Photonics Society and the International Society for Optics and Photonics. 
Xin Yin (S'06-M'09) received the Ph.D. degree in electronic engineering from Ghent University, Ghent, Belgium, in 2009. Since 2007, he has been a staff researcher with IMEC-INTEC and since 2013, he has been a Professor with the Department of Information Technology, Ghent University. He was and is active in European and International projects, such as PIEMAN, EUROFOS, MARISE, C3PO, DISCUS, PhoxTrot, MIRAGE, SPIRIT, WIPE, Teraboard, STREAMS, PICTURE, 5G-PHOS, and GreenTouch consortium. He has authored and coauthored more than 150 journal and conference publications in the field of high-speed electronics and fiber-optic communication. His research interests include high-speed opto-electronic circuits and transmitter/receiver subsystems, and low-power mixed-signal integrated circuit design for datacom/telecom $/ 5 \mathrm{G}$ applications. In 2014, he led a team including researchers from imec, Bell Labs USA/Alcatel-Lucent and Orange Labs, which won the GreenTouch 1000x award in recognition of the invention of the Bi-PON protocol and sustained leadership.

Joni Dambre (S'99-M'03) was born in Ghent, Belgium, in 1973. She received the degree in electrical engineering from Ghent University, Ghent, Belgium, in 1996, and the Ph.D. degree in computer science engineering from the Department of Electronics and Information Systems, Ghent University, in 2003. As a machine learning professor, she currently leads the UGent-imec AIRO research team (part of IDLab) addressing various topics related to machine learning, artificial and biological neural networks, and neuromorphic hardware.
Peter Bienstman (S'97-A'01-M'03) was born in Ghent, Belgium, in 1974. He received a degree in electrical engineering from Ghent University, Ghent, Belgium, in 1997, and the Ph.D. degree from the Department of Information Technology, Ghent University, in 2001, where he is currently a full-time Professor. He has published more than 110 papers and holds several patents. His research interests include several applications of nanophotonics (biosensors, photonic information processing, etc.) as well as nanophotonics modeling. He has been awarded an ERC starting grant for the Naresco-project: Novel paradigms for massively parallel nanophotonic information processing. 